\title{
Development of Contralaterally Controlled Functional Electrical Stimulation to Realize Multiple Grasping Postures with Data Glove
}

\author{
Soichi Takigawa ${ }^{1 *}$ and Hidenori Mimura ${ }^{1,2^{* *}}$ \\ ${ }^{1}$ Graduate School of Medical Photonics, Shizuoka University, \\ 3-5-1 Johoku, Naka-ku, Hamamatsu, Shizuoka 432-8011, Japan \\ ${ }^{2}$ Research Institute of Electronics, Shizuoka University, \\ 3-5-1 Johoku, Naka-ku, Hamamatsu, Shizuoka 432-8011, Japan
}

(Received August 24, 2021; accepted October 12, 2021)

Keywords: data globe, strain sensor, hand rehabilitation, functional electrical stimulation, multi-pad electrodes

To assist with functional electrical stimulation (FES) training for more effective recovery of grasping functions in paralyzed patients, our system recognizes multiple grasping postures of the opposite hand and selectively activates the muscles of the target hand using 24 multi-pad electrodes placed on the inner side of the forearm. Hand motion is detected via a data glove with carbon nanotube strain sensors placed at the metacarpophalangeal and proximal interphalangeal joints of each finger. The stimulating electrode patterns are established in advance by an automatic search of all electrode configurations. Multi-pad electrical stimulation based on the posture of the opposite hand symmetrically produces multiple grasping postures in the target hand.

\section{Introduction}

Biometric measurement and motion analysis are currently an area of active research. In particular, in the medical field, it is essential to have sensor systems that can collect data easily and non-invasively, without bothering the user. In this study, we have applied the data glove to functional electrical stimulation (FES) training.

FES is used to restore motor function in paralyzed patients because of stroke or spinal injury. ${ }^{(1)}$ In FES, electrical stimulations activate nerve tissue connected to muscle groups to contract muscles to induce movement of the hands or feet. ${ }^{(2)}$ Because hand function is critical in daily life, ${ }^{(3)}$ its restoration improves the quality of life of a paralyzed patient. ${ }^{(4,5)}$

Many studies have examined FES to restore hand movements in paralyzed patients. ${ }^{(6)}$ As an electrical stimulation method for the motor recovery of hemiplegic hand function after stroke, an electrical stimulation technique has been used to trigger movements of the non-paralyzed hand.(7) Contralaterally controlled functional electrical stimulation (CCFES) regulates the opening and closing of a paralyzed hand using the detection results of a data glove attached to 
the contralateral hand. ${ }^{(8)}$ Bilateral work in patients with chronic motor disorders after stroke allows the interhemispheric facilitation of a limb. ${ }^{(9)}$ Symmetrical movement reduces upper limb disability in some stroke patients. ${ }^{(10-12)}$ CCFES has been reported to be more advantageous than cyclic electrical stimulation for motor function recovery. ${ }^{(13,14)}$ In addition, a method combining FES triggered by myoelectricity associated with the movement of the non-paralyzed hand and mirror therapy has been proposed. ${ }^{(15,16)}$ In mirror therapy, a mirror is placed between the paralyzed and non-paralyzed limbs of a subacute stroke patient. Visual feedback of nonparalyzed hand movements using a mirror is a well-documented technique to restore the function of a paralyzed hand. ${ }^{(17)}$ Studies have shown that a combination of visual feedback and FES is more effective than FES or mirror therapy alone. ${ }^{(15,16)}$ These methods use the movement of the opposite hand as a trigger for electrical stimulation. However, the small number of stimulating electrodes limits the movement pattern of the paralyzed limb by electrical stimulation at present.

FES using multi-pad electrodes with an array of non-invasive electrodes has been proposed to selectively produce muscle contraction in a paralyzed hand. ${ }^{(18)}$ Multi-pad electrodes arranged in an array can dynamically adapt the area and position of the applied stimulus. The stimulation area can be changed without repositioning the electrodes. Consequently, muscle contraction can be efficiently and selectively induced.

Many FES methods using multiple electrodes have been examined to select the optimal stimulation electrode. One method selects stimulation electrodes to produce the desired hand posture based on a visual inspection of the muscle response by a medical professional. ${ }^{(19,20)}$ Because this method requires the presence of a medical professional, patients cannot train themselves in a home environment. Other studies have proposed methods to automatically search for the optimal stimulation point without a visual inspection by a medical professional. The search for the optimal stimulation point is performed by detecting the kinematic response provoked by an electrical stimulation and comparing it with predefined movements. ${ }^{(21,22)}$

Several types of devices have been adopted to detect the kinematic response induced by electrical stimulation. Examples include depth cameras; ${ }^{(23,24)}$ optical motion capture systems; ${ }^{(25)}$ goniometers; ${ }^{(21,23)}$ flex sensors; ${ }^{(26,27)}$ gyro, acceleration, and other motion sensors; ${ }^{(28,29)}$ and load cells. ${ }^{(30)}$ These devices have their own challenges. For example, the camera setup is burdensome. Additionally, blind spots make it difficult to capture the hand and the positional relationship between the camera and the hand. Although widely adopted, motion sensors such as goniometers, flex sensors, and gyro-accelerometers are complex systems. Attaching them to a paralyzed hand is arduous, and reproducibility is problematic even in a clinical setting. A different approach to detect electrophysiological responses caused by electrical stimulation uses electromyography (EMG) of the forearm. ${ }^{31,32)}$ This method can detect responses to low levels of stimulation. However, each electrode must be attached separately, limiting the placement of the stimulation electrodes. Adapting the multi-pad electrodes to CCFES or a method that combines mirror therapy and FES should realize motions beyond simple grasping such as bending of the fingers independently. The data gloves have been adopted to detect the kinematic response of each finger induced by electrical stimulation with multi-pad electrodes. ${ }^{(33,34)}$ However, methods to detect and recognize motions of the opposite hand and methods to provide electrical stimulation with multi-pad electrodes to produce the corresponding hand postures in the target hand have not been sufficiently studied. 
In this study, we propose an FES training method triggered by motions of the opposite hand. Symmetrical motions in the target hand are triggered by the response to multiple motions of the opposite hand. The posture of the opposite hand is recognized by a data glove, and the electrical stimulation points of the multi-pad electrodes of the target hand are dynamically selected on the basis of the recognized posture. The patient can make the target hand produce postures symmetrical to the multiple grasping postures of the opposite hand without being aware of a special device. Electrical stimulation points that elicit the desired grasping motions are explored in advance with reference to the postures of the opposite hand. The proposed method can be applied to CCFES and a combined method of mirror therapy and FES to train paralyzed patients to recover their grasping function more effectively.

\section{Materials and Methods}

The system consisted of multichannel FES and the data glove. These devices were controlled by PC application software built in Microsoft Visual C\#. Figure 1 shows a block diagram of multichannel FES. It was composed of a power supply, microcontroller unit (MCU), constant current circuit, drive electrode selectors, and multi-pad electrodes. We used eight nickel metal hydride (Ni-MH) batteries (1.2 V, $2400 \mathrm{mAh}$ ) connected in series to prevent electric shock from the power supply. The power supply was connected to a constant current circuit and the MCU (Teensy 3.2, 32-bit ARM Cortex-M4 processor). The microcontroller was connected to a host PC via a USB isolator to protect subjects from electric shock. The microcontroller selects the stimulation electrodes and controls the pulse signals based on the control signals from the PC application via a USB every $100 \mathrm{~ms}$. The constant current circuit consisted of a boost-type DCDC converter and a constant current source. The DC-DC converter connected to the power supply boosted the voltage from the Ni-MH battery to $50 \mathrm{~V}$ and supplied power to the constant current source. The constant current source was configured in the range of $0-50 \mathrm{~mA}$ in $1 \mathrm{~mA}$ increments.

The drive electrode switching circuit consisted of a shift register and a switching transistor array. The shift register was controlled by the MCU via serial peripheral interface

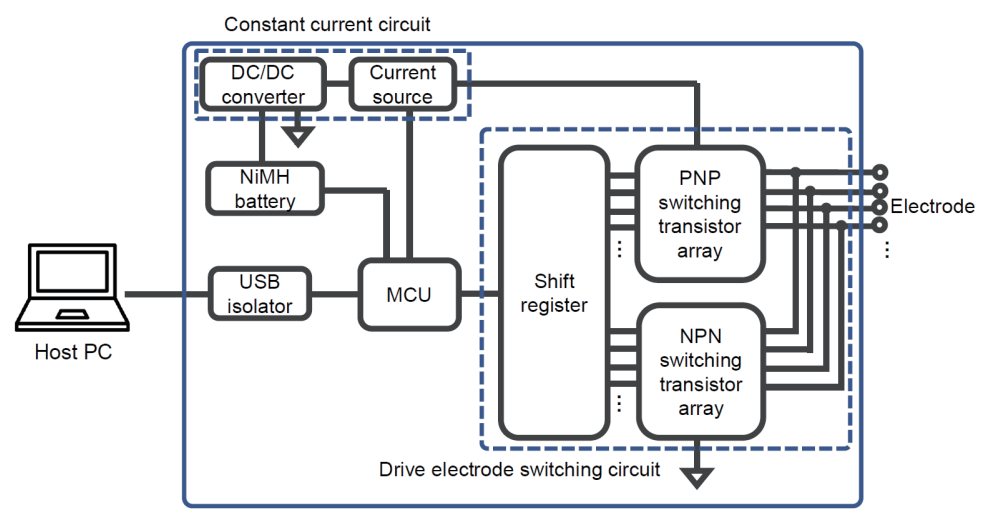

Fig. 1. (Color online) Block diagram of multichannel FES. 
communication. The output of the shift register was connected to the switching transistor, which modulated the current supplied by the constant current source and applied it to the selected electrode. By configuring a multichannel H-bridge circuit with a drive electrode switching circuit and electrode pads, a maximum of 32 electrodes were set up as either active or return electrodes.

Figure 2(a) shows the belt-shaped multi-pad electrodes and Fig. 2(b) shows multi-pad electrodes setup to visualize the target arm. The multi-pad electrodes consisted of wet gel electrodes (Omron Corporation, HV-LLPAD). Each electrode was $20 \mathrm{~mm}$ wide, $30 \mathrm{~mm}$ long, and $24 \mathrm{~mm}$ pitch. Up to eight gel electrodes could be fixed with a hook-and-loop fastener belt in one row along the short side direction. In this experiment, six electrodes were aligned on one belt. The experiment employed four belts, which resulted in a total of 24 electrodes placed on the inner side of the forearm. They were not affected by the contact resistance between the electrodes and the body because current-controlled signals were applied to the electrodes.

To measure the finger posture of the left and right hands, we used the data glove. The data glove was jointly developed by Shizuoka University and Yamaha Corporation. ${ }^{(35)}$ Figure 3(a) shows the data glove with multiple carbon nanotube (CNT) strain sensors in place and Fig. 3(b) shows the layout of the CNT strain sensors. The data glove has CNT strain sensors at 11 different locations on the carpometacarpal (CMC), metacarpophalangeal (MP), and interphalangeal (IP)

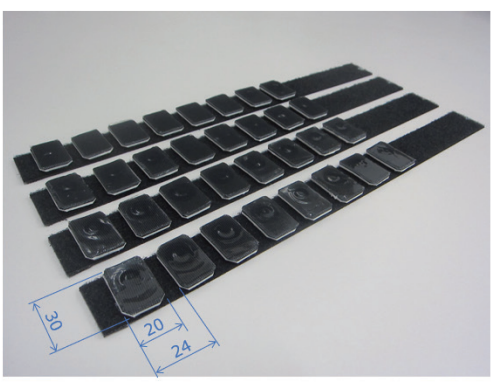

(a)

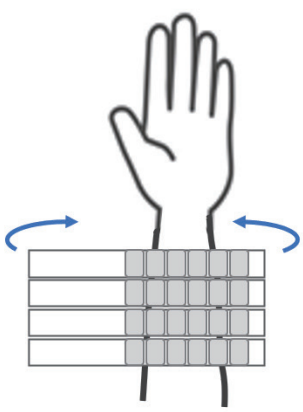

(b)

Fig. 2. (Color online) (a) Belt-shaped multi-pad electrodes and (b) multi-pad electrodes set up to visualize the target arm.

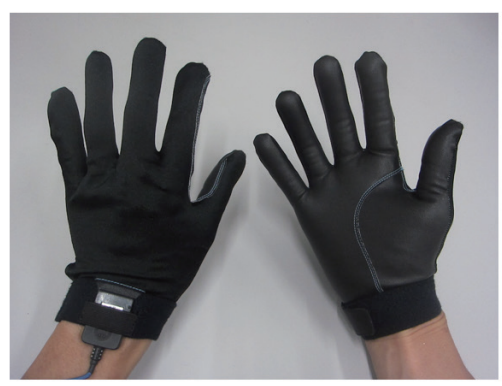

(a)

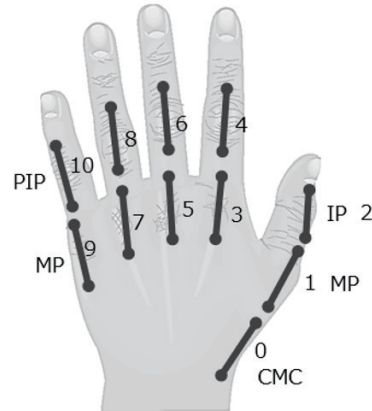

(b)

Fig. 3. (Color online) (a) Data glove with multiple CNT strain sensors in place and (b) layout of the CNT strain sensors. 
joints of the first finger and the MP and proximal interphalangeal (PIP) joints of the second through the fifth fingers. The CNT strain sensor is composed of CNT sheets ${ }^{(36,37)}$ and an elastomer material, and its resistance changes according to the amount of stretching. As a finger joint bends, the CNT strain sensor stretches and the resistance increases. In contrast, as the finger joint stretches, the sensor shrinks and the resistance decreases. Consequently, the degree of bending of each joint is individually detected. The strain on the fingers is minimal because the CNT strain sensor is very thin and adheres to the human skin surface. Furthermore, the data glove can be worn comfortably for a long time because of the breathable fabric.

The data glove was connected to the PC via a USB. The PC recorded the measured resistance of each sensor every $100 \mathrm{~ms}$ with 12-bit resolution. The resistance $\boldsymbol{R}_{\boldsymbol{j}}$ of each sensor was calibrated using the resistance $\boldsymbol{R}_{\boldsymbol{j}_{\text {closed }}}$ of the closed hand posture and the resistance $\boldsymbol{R}_{\boldsymbol{j}_{\text {open }}}$ of the relaxed open posture to define the rate of flexion $\boldsymbol{P}_{\boldsymbol{j}}$ of each joint as follows.

$$
\boldsymbol{P}_{\boldsymbol{j}}=\frac{\boldsymbol{R}_{\boldsymbol{j}}-\boldsymbol{R}_{\boldsymbol{j}_{\text {open }}}}{\boldsymbol{R}_{\boldsymbol{j}_{\text {open }}}-\boldsymbol{R}_{\boldsymbol{j}_{\text {closed }}}}
$$

In this study, the sensor placed in the CMC of the first finger was not used. Thus, $j$ was from 1 to 10, as shown in Fig. 3. The experiment was conducted with four healthy male subjects in their 30s to 50s. This study was performed in accordance with the Declaration of Helsinki and was approved by the Research Ethics Committee of Shizuoka University in compliance with the Regulations on Research Involving Human Subjects at Shizuoka University. The methods, hazards, and benefits of the experiment were explained to the subjects and their consent was obtained prior to the experiment. All subjects were right-handed.

In the experiment, the right hand was the opposite hand, which presented the grasping posture. The left hand was the target hand to which the electrical stimulation was applied. The subjects sat in a chair during the experiment with their elbows bent. The left and right forearms were placed on a desk with palms facing up. The subjects wore data gloves on their left and right hands to measure finger postures. Then 24 gel electrodes placed on four belts were attached to the inside of the left forearm for electrical stimulation. In addition, hand motions during the experiment were recorded with a video camera and analyzed. The subjects were asked not to resist the motions evoked by the electrical stimulation.

Figure 4 shows the experimental flow (upper) and the target grasping postures and a relaxed open posture recorded with the opposite hand (lower). The experiment was conducted as follows. In step 1, multiple grasping postures were recorded with the subject's opposite (right) hand using the data glove to create a data table for identifying hand postures. In step 2, the stimulation electrodes of the subject's target hand were scanned, and the optimal stimulation electrodes to produce the pre-recorded target grasping postures were determined by detecting the evoked postures with the data glove. In step 3, symmetrical postures were produced in the target (left) hand. Specifically, the grasping posture of the opposite hand was detected with the data glove. Then the optimal electrical stimulation pattern identified in the search was applied to the target hand according to the discriminated grasping posture. This procedure produced symmetrical postures in the target hand in response to the grasping postures of the opposite hand. 


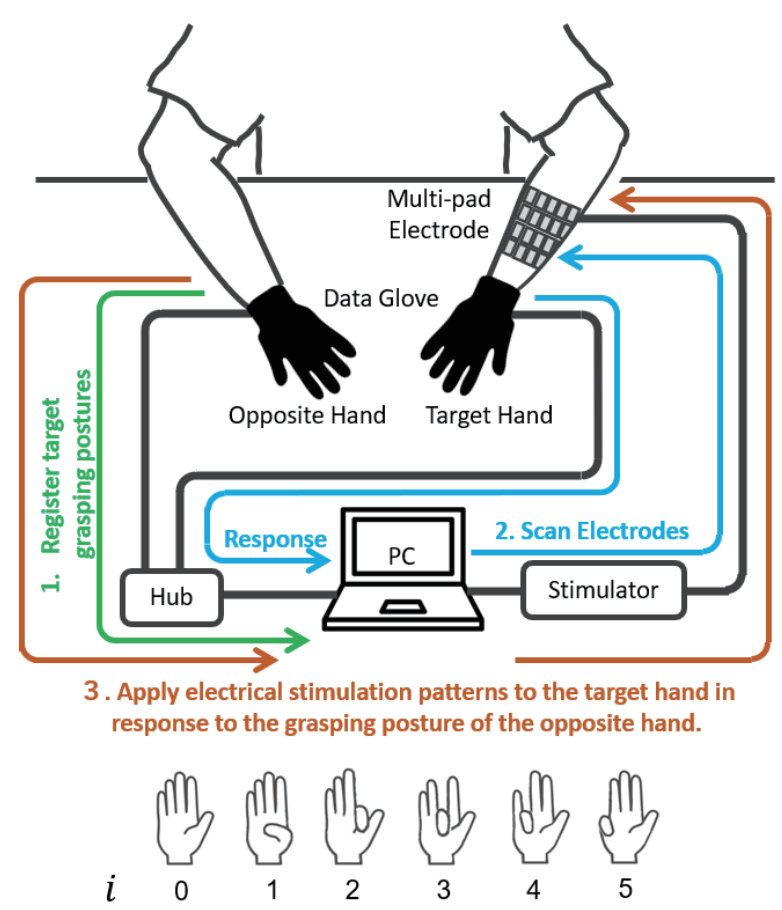

Fig. 4. (Color online) Flow of the three steps of the experiment (upper) and target grasping postures and a relaxed open posture recorded with the opposite hand (lower).

\subsection{Registering target grasping postures with opposite hand (step \#1)}

Six different postures were recorded: five grasping postures in which one finger was bent and a relaxed open hand posture (Fig. 4). $\boldsymbol{P}_{\boldsymbol{O} i j}(i=0-5, j=1-10)$ is the output of strain sensor $j$ of the data glove in hand posture pattern $i$. The output of the data glove in hand posture pattern $i$ is pattern vector $\overrightarrow{\boldsymbol{P}_{\boldsymbol{i}}}(i=0-5)$. The output pattern vectors of the data glove for each hand posture were memorized as a data table. This table was used to evaluate the hand postures in the search for stimulation electrodes for the target hand and to recognize the hand postures of the opposite hand.

\subsection{Identifying optimal stimulation electrodes by scanning (step \#2)}

We searched for the electrical stimulation patterns in the target hand, which produce postures symmetrical to the grasping postures registered with the opposite hand. To apply a stimulus, all electrodes in the pattern and one of the remaining electrodes were set as the active and return electrodes, respectively. The electrode combinations were automatically set by the PC application. Then hand postures produced by the electrical stimulation were recorded by the data glove and video camera. The electrical stimulations were biphasic pulses with a period of $30 \mathrm{msec}$ and a pulse width of $200 \mu \mathrm{s}$. The applied current was set within a range so that the subject did not feel uncomfortable. The duration of the electrical stimulation was $1 \mathrm{~s}$ for each 
pair of electrodes. The time interval when changing the electrode combination was $1 \mathrm{~s}$. If an electrode made a subject feel discomfort, it was excluded from the combination during the search by changing the settings of the PC application.

Using the data detected by the data glove, we evaluated the hand postures produced by the electrical stimulation for each electrode combination and look-up tables were created. A custom script written in Python was used to evaluate the data and create the look-up tables. For each electrode combination, the hand posture at $1 \mathrm{~s}$ after the start of electrical stimulation was defined as the hand posture produced by that electrode combination. The output of strain sensor $j$ of the data glove in combination $n$ of the stimulus and return electrodes was defined as $\boldsymbol{P}_{\boldsymbol{T} \boldsymbol{n} \boldsymbol{j}}(n=1-522, j$ $=1-10$ ), and the output vector of the data glove in combination $n$ of the stimulus and return electrodes was $\overrightarrow{\boldsymbol{P}_{\boldsymbol{n}}}(n=1-522)$.

To evaluate the similarity between the pattern vector $\overrightarrow{\boldsymbol{P}_{\boldsymbol{n}}}$ in stimulus and return electrode combination $n$ and the pattern vectors $\overrightarrow{\boldsymbol{P}_{\boldsymbol{O} \boldsymbol{i}}}(i=0-5)$ of the respective target grasping postures recorded by the opposite hand, we defined an evaluation function $\boldsymbol{R}_{\boldsymbol{i n}}$ on the basis of the normalized Euclidean distance ${ }^{(38)} \boldsymbol{d}_{\boldsymbol{n}}\left(\overrightarrow{\boldsymbol{P}_{\boldsymbol{O}}}, \overrightarrow{\boldsymbol{P}_{\boldsymbol{T} \boldsymbol{n}}}\right)$ as

$$
\boldsymbol{R}_{\boldsymbol{i n}}=1-\boldsymbol{d}_{\boldsymbol{n}}\left(\overrightarrow{\boldsymbol{P}_{\boldsymbol{O} i}}, \overrightarrow{\boldsymbol{P}_{\boldsymbol{T n}}}\right)=1-\sqrt{\left(\overrightarrow{\boldsymbol{P}_{\boldsymbol{O}}}-\overrightarrow{\boldsymbol{P}_{\boldsymbol{T n}}}\right)^{2} / \boldsymbol{N}}
$$

where $N=10$ is the number of effective strain sensors. The optimal electrode for producing grasping posture pattern $i$ was the combination of the stimulus and return electrodes with the largest $\boldsymbol{R}_{\boldsymbol{i n} \boldsymbol{n}}$. Electrode combinations that caused large wrist motions or did not produce grasping motions were manually excluded from the look-up table, and the electrode combinations were adopted in order of $\boldsymbol{R}_{\boldsymbol{i n}}$.

\subsection{Applying electrical stimulation patterns to target hand in response to grasping posture of opposite hand (step \#3)}

The similarity between the output vector $\overrightarrow{\boldsymbol{P}_{\boldsymbol{O}}}$ of the data glove for the opposite hand posture and the pattern vector $\overrightarrow{\boldsymbol{P}_{\boldsymbol{O}} \boldsymbol{i}}(i=0-5)$ of each hand posture recorded in step \#1 was calculated in real time. The electrical stimulation pattern corresponding to the grasping posture pattern with the highest similarity was applied to the target hand on the basis of the look-up table created in step \#2. For pattern recognition of the hand posture of the opposite hand, we used evaluation function $\boldsymbol{R}_{\boldsymbol{i}}(i=0-5)$ based on the normalized Euclidean distance, which is given as

$$
\boldsymbol{R}_{\boldsymbol{i}}=1-\boldsymbol{d}_{\boldsymbol{n}}\left(\overrightarrow{\boldsymbol{P}_{\boldsymbol{O}}}, \overrightarrow{\boldsymbol{P}_{\boldsymbol{O}}}\right)=1-\sqrt{\left(\overrightarrow{\boldsymbol{P}_{\boldsymbol{O} i}}-\overrightarrow{\boldsymbol{P}_{\boldsymbol{O}}}\right)^{2} / \boldsymbol{N}}
$$

The hand posture pattern $i$ with the largest $\boldsymbol{R}_{\boldsymbol{i}}$ was defined as the hand posture of the opposite hand at time $t$. 


\section{Results}

Firstly, the five target grasping postures and the relaxed open posture were presented with the opposite hand. The output of the data glove for each hand posture was recorded. Then, a search for electrical stimulation patterns that produced the five recorded target grasping postures was executed with the target hand. One of the 24 electrodes was used as the active electrode, and 23 different return electrodes were combined with it, giving a total of 552 combinations of stimulus patterns. The search for the stimulation patterns took about $20 \mathrm{~min}$. Subject D showed discomfort with the stimulation at some electrodes. Thus, the corresponding electrodes were excluded from the search. The optimal electrodes to produce the five target grasping postures were automatically identified by the search algorithm, and combinations of electrodes that caused a large wrist motion or did not well reproduce the grasping postures were manually excluded from the search results.

Figure 5 shows the stimulation electrodes identified to produce the five target grasping postures by subject. In three subjects, suitable electrodes were identified to produce all five target postures. However, for subject D, electrodes suitable to induce four target grasping postures were identified, but the middle finger did not produce a flexion motion on its own. The optimal electrode patterns for each grasping motion identified by the search algorithm significantly differed by subject. From the search results, a look-up table of the electrode patterns that produce the target grasping posture was created for each subject.

Finally, we confirmed that the target hand produced grasping postures symmetrical to the opposite hand by applying the electrical stimulation pattern using the look-up table created with the search algorithm. The postures of the opposite hand, which triggered the electrical stimulation, were detected in real time by the data glove and evaluated using evaluation functions for each target posture pattern recorded in advance.

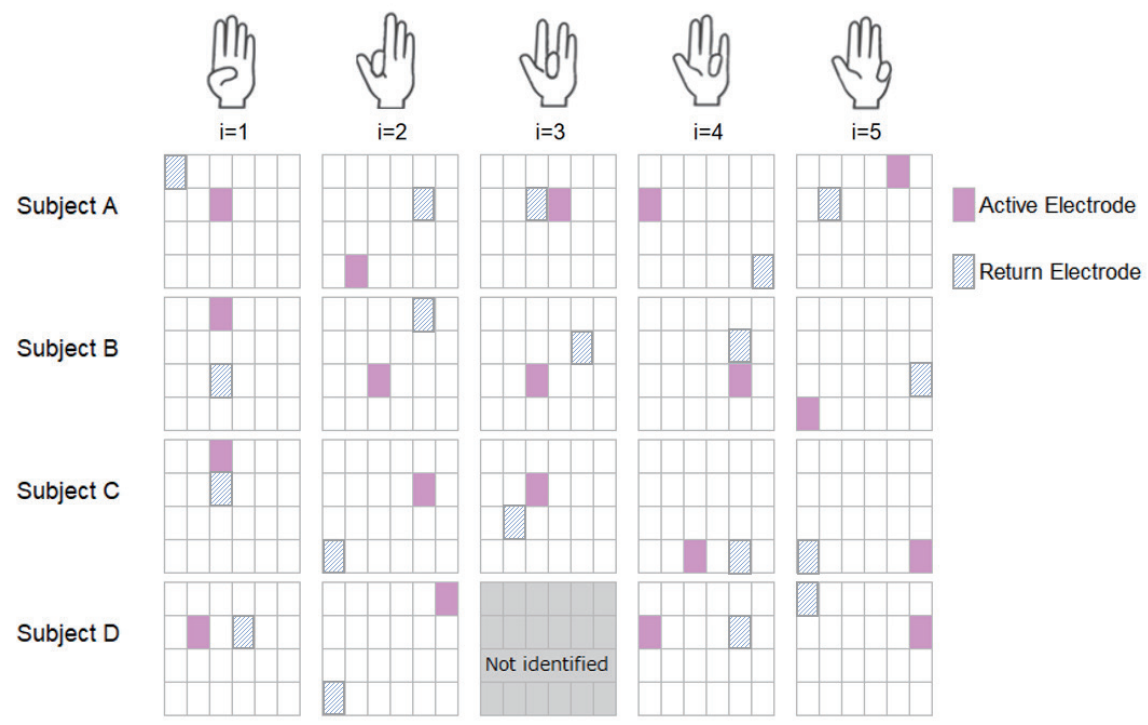

Fig. 5. (Color online) Stimulation electrodes identified to produce the five target grasping postures for each subject. 
Figure 6 shows the exemplary motion of each joint, the evaluation function of each posture pattern of the opposite hand at that time, and the motion of each joint of the target hand induced by electrical stimulation when subject A bent only the index finger of the opposite hand. In this case, the MP and PIP rates of flexion of the index finger increased. The evaluation function value of posture pattern $i=2$ increased as the flexion rates of MP and PIP increased, while that of posture pattern $i=0$ decreased. Once the evaluation function value of posture pattern $i=2$ was maximized compared with the other posture patterns, the electrode for posture pattern $i=2$ was selected using the look-up table, and electrical stimulation was applied to the target hand. The electrical stimulation applied to the subject's target hand produced a symmetrical motion, and the MP and PIP flexion rates of the index finger of the subject's hand increased. Upon relaxing the opposite hand, the MP and PIP flexion rates decreased. The evaluation function value of posture pattern $i=2$ decreased with the decrease in the MP and PIP flexion rates, while that of posture pattern $i=0$ increased. When the evaluation function value for posture pattern $i=$ 0 was maximized relative to those for the other postures, the electrical stimulation applied to the target hand was stopped.

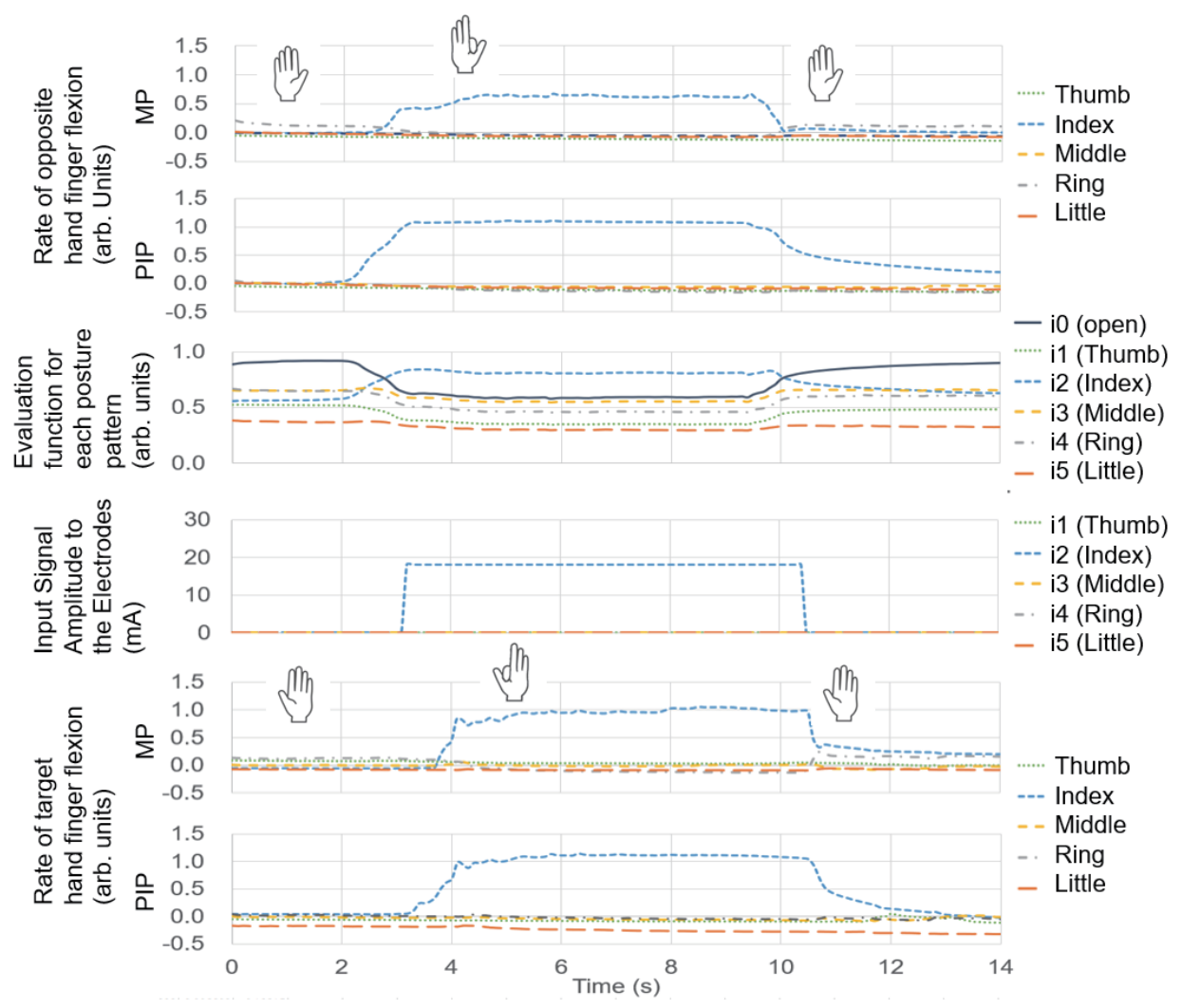

Fig. 6. (Color online) Grasping motion of the index finger of the target hand caused by electrical stimulation in response to the posture of the opposite hand of subject A. Flexion rates of the finger joints (MP, PIP) of the opposite hand calculated using Eq. (1) are plotted in the first and second graphs. Evaluation functions for every target posture calculated using Eq. (3) from the flexion rate of the finger joints of the opposite hand are plotted in the third graph. Input signal current amplitude to the electrodes for every target posture are plotted in the fourth graphs. The last two graphs show the rate of flexion of the finger joints (MP, PIP) of the target hand calculated using Eq. (1) when the electrodes were activated, as derived from the look-up table based on the evaluation function. 
Figure 7 shows the postures presented by the opposite hand and the postures of the target hand produced by the electrical stimulation selected in response to the posture of the opposite hand. In all subjects, symmetrical postures were produced in the target hand in response to multiple posture patterns of the opposite hand. However, subject D did not produce posture 3 because the electrode search algorithm did not identify the appropriate electrode stimulation.

\section{Discussion}

In this study, we have proposed a method to search for electrical stimulation points that produce multiple grasping postures in FES using multi-pad electrodes and to produce symmetrical postures in the target hand in response to those of the opposite hand. The postures of the opposite hand were detected by the data glove, the stimulation electrodes were dynamically selected in response to the detected hand postures, and the electrical stimulation was applied to the target hand. The subjects were able to express multiple grasping motions in the target hand by presenting the target grasping posture with the opposite hand without operating any switches.

In the proposed method, each electrode of the multi-pad electrodes was assigned to an active electrode or a return electrode, which selectively increased the patterns of current paths and activated the muscles. This realized independent grasping motions of each finger, which can be attributed to the increased path pattern of current flow. ${ }^{(35)}$ The optimal electrode patterns for each grasping posture identified by this search method significantly differed by subject. Therefore, the search algorithm for the stimulation electrodes must be applied to each subject at least once.

On the other hand, it has been reported that the optimal stimulation positions for each patient are reproducible. ${ }^{(29)}$ In this study, the multi-pad electrode was composed of multiple belt-shaped electrode arrays. By integrating the electrode arrays and configuring the multi-pad electrodes with reproducibility by attaching them to the subject, the second search process may be omitted.

In addition, some subjects were not able to achieve specific grasping motions. This may be due to the size of the individual electrodes. If the electrode is too large, the resolution of the

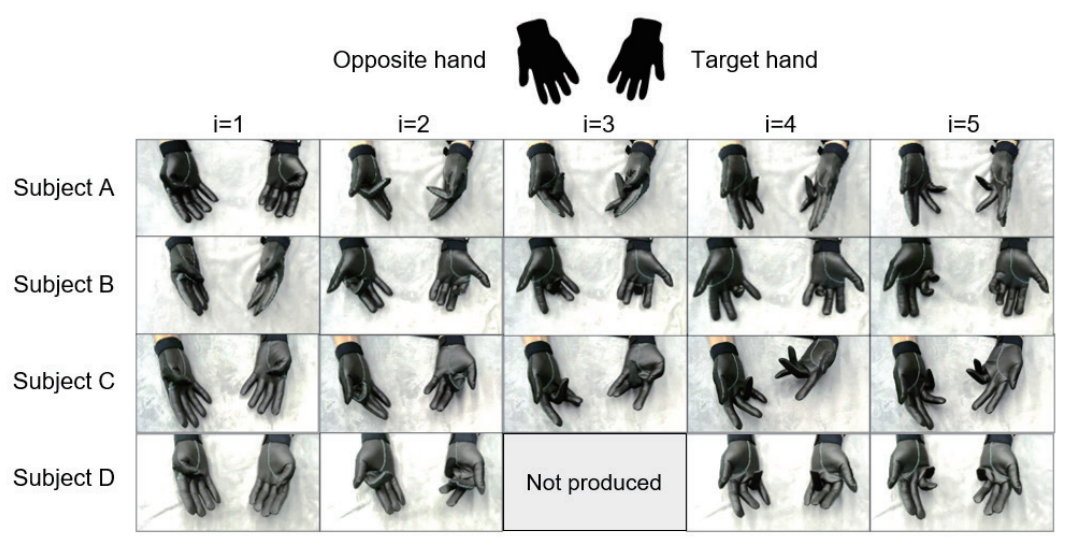

Fig. 7. (Color online) Postures of the opposite hand and postures of the target hand produced by the electrical stimulation in response to the posture of the opposite hand of each subject. 
stimulation points may be insufficient. Another reason may be inadequate search points. If a subject showed discomfort with specific electrodes during the electrode search, they were excluded from the search. Additionally, we manually excluded electrode combinations with large wrist motions from the look-up table created with the search algorithm. By placing the device so that it also detects the posture at the wrist and adding a posture parameter of the wrist to the pattern vector used in the evaluation function, electrode combinations with large wrist motions can be excluded in an automatic search. ${ }^{(29,31,34)}$ We showed the results of one trial for each subject. We plan to show some statistical verification such as successful rates of shaping hand forms in a future study. Finally, we employed a glove-shaped device to detect the motion of the target hand when applying an electrical stimulation. Since wearing a glove-like device on a paralyzed hand may be difficult, we plan to develop an easier-to-wear device in the future.

\section{Conclusions}

We have developed a system that combines multichannel FES and a data glove with CNT strain sensors. Experiments on four healthy subjects demonstrated that the system selectively activates the muscles of the target hand in response to the grasping postures of the opposite hand. Additionally, the method produces postures symmetrical to those of the opposite hand. By this method, patients can intuitively train multiple grasping motions using FES without operating special instruments. Applying this method to CCFES or its combination with mirror therapy should improve the efficacy of training with FES.

\section{Acknowledgments}

The authors wish to thank Prof. Toru Aoki, Prof. Shigeki Tani, and Dr. Katsunori Suzuki for assistance in designing the study.

\section{References}

1 P. H. Peckham and J. S Knutson: Annu. Rev. Biomed. Eng. 7 (2005) 1. http://doi.org/10.1146/annurev. bioeng.6.040803.140103

2 S. Micera, T. Keller, M. Lawrence, M. Morari, and D. B. Popovic: IEEE Eng. Med. Biol. Mag. 29 (2010) 3. https://doi.org/10.1109/MEMB.2010.936547

3 M. T. Duruöz: Hand function (Springer, New York, 2014). https://doi.org/10.1007/978-1-4614-9449-2

4 M. L. Niemi, R. Laaksonen, M. Kotila, O. Waltimo: Stroke 19 (1988) 9. http://doi.org/10.1161/01.STR.19.9.1101

5 K. Laurent, M. P. De Sèze, C. Delleci, M. Koleck, P. Dehail, J. M. Orgogozo, and J. M. Mazaux: Ann. Phys. Rehabil. Med. 54 (2011) 6. http://doi.org/10.1016/j.rehab.2011.06.002

6 F. Quandt and F. C. Hummel: Exp. Transl. Stroke Med. 6 (2014) 9. http://doi.org/10.1186/2040-7378-6-9

7 J. S. Knutson, M. J. Fu, L. R. Sheffler, and J. Chae: Phys. Med. Rehabil. Clin. N. Am. 26 (2015) 4. http://doi. org/10.1016/j.pmr.2015.06.002

8 J. S. Knutson, M. Y. Harley, T. Z. Hisel, and J. Chae: Arch. Phys. Med. Rehabil. 88 (2007) 4. http://doi. org/10.1016/j.apmr.2007.01.003

9 A. R. Luft, S. McCombe-Waller, J. Whitall, L. W. Forrester, R. Macko, J. D. Sorkin, J. B. Schulz, A. P. Goldberg, and D. F. Hanley: Am. Med. Assoc. 292 (2004) 15. http://doi.org/10.1001/jama.292.15.1853

10 K. Lin, Y. Chen, C. Chen, C. Wu, and Y. Chang: Neurorehabil. Neural Repair 24 (2010) 1. http://doi. org $/ 10.1177 / 1545968309345268$

11 R. Sleimen-Malkoun, J. J. Temprado, L. Thefenne, and E. Berton: BMC Neurol. January 11 (2011). https://doi. 
org/10.1186/1471-2377-11-11

12 J. Metrot, D. Mottet, I. Hauret, L. van Dokkum, H.-Y. Bonnin-Koang, K. Torre, and I. Laffont: Neurorehabil. Neural Repair 27 (2013) 3. http://doi.org/10.1177/1545968312461072

13 J. S. Knutson, M. Y. Harley, T. Z. Hisel, S.D. Hogan, M. M. Maloney, and J. Chae: Neurorehabil. Neural Repair 26 (2012) 3. http://doi.org/10.1177/1545968311419301

14 J. S. Knutson, D. D. Gunzler, R. D. Wilson, and J. Chae: Stroke 47 (2016). http://doi.org/10.1161/ STROKEAHA.116.013791

15 J. H. Kim and B. H. Lee: Occup. Ther. Int. 22 (2015) 2. http://doi.org/10.1002/oti.1384

16 G. J. Yun, M. H. Chun, J. Y. Park, and B. R. Kim: Ann. Rehabil. Med. 35 (2011) 3. http://doi.org/10.5535/ arm.2011.35.3.316

17 G. Yavuzer, R. Selles, N. Sezer, S. Sütbeyaz, J. B. Bussmann, F. Köseog lu, M. B. Atay, and H. J. Stam: Arch. Phys. Med. Rehabil. 89 (2008) 393. http://doi.org/10.1016/j.apmr.2007.08.162

18 A. D. Koutsou, J. C. Moreno, A. J. del Ama, E. Rocon, and J. L. Pons: J. NeuroEng. Rehabil. 13 (2016) 1. http:// doi.org/10.1186/s12984-016-0165-2

19 J. Malešević, M. Štrbac, M. Isaković, V. Kojić, L. Konstantinović, A. Vidaković, S. Dedijer Dujović, M. Kostić, and T. Keller: Artif. Organs 41 (2017) 11. http://doi.org/10.1111/aor.13057

20 C. Salchow-Hömmen, N. Jankowski, M. Valtin, L. Schönijahn, S. Böttcher, F. Dähne, and T. Schauer: J. NeuroEng. Rehabil. 15 (2018) 1. http://doi.org/10.1186/s12984-018-0460-1

21 D. B. Popović and M. B. Popović: J. Neurosci. Methods 178 (2009) 1. http://doi.org/10.1016/j. jneumeth.2008.12.003

22 S. B. O'Dwyer, D. T. O’Keeffe, S. Coote, and G. M. Lyons: Med. Eng. Phys. 28 (2006) 2. http://doi.org/10.1016/j. medengphy.2005.03.010

23 K. L. Meadmore, T. A. Exell, E. Hallewell, A. M. Hughes, C. T. Freeman, M. Kutlu, V. Benson, E. Rogers, and J. H. Burridge: J. NeuroEng. Rehabil. 11 (2014) 1. http://doi.org/10.1186/1743-0003-11-105

24 M. Kutlu, C. T. Freeman, E. Hallewell, A. M. Hughes, and D. S. Laila: Med. Eng. Phys. 38 (2016) 4. http://doi. org/10.1016/j.medengphy.2016.01.004

25 A. Crema, N. Malesevic, I. Furfaro, F. Raschella, A. Pedrocchi, S. Micera: IEEE Trans. Neural Syst. Rehabil. Eng. 26 (2018) 2. http://doi.org/10.1109/TNSRE.2017.2703151

26 N. Malešević, L. Maneski, V. Ilić, N. Jorgovanović, G. Bijelić, T. Keller, D. Popović: J. NeuroEng. Rehabil. 9 (2012) 1. http://doi.org/10.1186/1743-0003-9-66

27 O. Schill, R. Rupp, C. Pylatiuk, S. Schulz, and M. Reischl: IEEE Toronto Int. Conf. Sci. Technol. Humanity (IEEE, 2009). http://doi.org/10.1109/TIC-STH.2009.5444408

28 N. Malesevic, L. Popovic, G. Bijelic, and G. Kvascev: J. Autom. Control 20 (2010) 1. http://doi.org/10.2298/ JAC1001053M

29 A. D. Koutsou, E. Rocon, F. Brunetti, J. C. Moreno, and J. L. Pons: A novel method for the analysis of forearm muscle activation by selective sFES. 18th IFESS Annu. Conf. 2013.

30 A. J. Westerveld, A. C. Schouten, P. H. Veltink, and H. van der Kooij: IEEE Trans. Neural Syst. Rehabil. Eng. 20 (2012) 1. http://doi.org/10.1109/TNSRE.2011.2178749

31 C. De Marchis, T. S. Monteiro, C. Simon-Martinez, S. Conforto, and A. Gharabaghi: J. NeuroEng. Rehabil. 13 (2016) 1. http://doi.org/10.1186/s12984-016-0129-6

32 H. Shin, Y. Zheng, and X. Hu: Stimulation Front. Neurol. 9 (2018). http://doi.org/10.3389/fneur.2018.01101

33 K. Yang, C. Freeman, R. Torah, S. Beeby, and J. Tudor: Sens. Actuators, A 213 (2014) 108. http://doi. org/10.1016/j.sna.2014.03.025

34 E. Imatz-Ojanguren, E. Irigoyen, D. Valencia-Blanco, and T. Keller: Med. Eng. Phys. 38 (2016) 1214. https:// doi.org/10.1016/j.medengphy.2016.06.008

35 K. Suzuki, K. Yataka, Y. Okumiya, S. Sakakibara, K. Sako K, H. Mimura, and Y. Inoue: Am. Chem. Soc. Sens. 1 (2016) 6. http://doi.org/10.1021/acssensors.6b00145

36 Y. Inoue, K. Kakihata, Y. Hirono, T. Horie, A. Ishida, and H. Mimura: Appl. Phys. Lett. 92 (2008) 21. http://doi. org/10.1063/1.2937082

37 Y. Inoue, Y. Suzuki, Y. Minami, J. Muramatsu, Y. Shimamura, K. Suzuki, M. A. Ghemes, M. Okada, S. Sakakibara, and H. Mimura: Carbon 49 (2011) 7. http://doi.org/10.1016/j.carbon.2011.02.010

38 M. M. Deza, and E. Deza: Encyclopedia of Distances (Springer, Berlin, Heidelberg, 2009). http://doi. org/10.1007/978-3-642-00234-2 